\title{
Digital Models: Comparison of Scanning Angulations and Superimposition
}

\author{
Bruno Nehme Barbo, Fabiane Azeredo, Luciane Macedo de Menezes* \\ School of Health and Life Sciences, Pontifical Catholic University of Rio Grande do Sul (PUCRS), Porto Alegre, Brazil \\ Email: bruno.barbo@hotmail.com, fabianeazeredo@hotmail.com, ^luciane.menezes@pucrs.br
}

How to cite this paper: Barbo, B.N., Azeredo, F. and de Menezes, L.M. (2020) Digital Models: Comparison of Scanning Angulations and Superimposition. Open Journal of Stomatology, 10, 218-229.

https://doi.org/10.4236/ojst.2020.108021

Received: June 26, 2020

Accepted: July 31, 2020

Published: August 3, 2020

Copyright $\odot 2020$ by author(s) and Scientific Research Publishing Inc. This work is licensed under the Creative Commons Attribution International License (CC BY 4.0).

http://creativecommons.org/licenses/by/4.0/

Open Access

\begin{abstract}
Background: The aim of this study was to evaluate linear measurements in digital models, scanned at different angulations, in order to compare four methods of superimposing digital models. Methods: Dental models obtained from the database of the School of Health and Life Sciences of the Pontifical Catholic University of Rio Grande do Sul (PUCRS) were scanned three-dimensionally at different angulations $\left(0^{\circ}, 5^{\circ}, 10^{\circ}\right.$ and $15^{\circ}$ in relation to the occlusal plane) and divided in four groups. The sample size was calculated and 43 dental models were used in this study. Linear measurements were obtained to assess the central and lateral portions of the 3D models, so that any possible distortion would be detected. Additionally, the digital models were superimposed using four approaches based on anatomical references: Methods 1 (three central landmarks), 2 (three lateral landmarks), 3 (surface + single landmark) and 4 (surface + three landmarks). Intraclass correlation coefficient was applied to evaluate the intra-observer reliability. Kruskal-Wallis was calculated to compare the groups according to the angulation during scanning, and Kappa to evaluate the agreement between superimposition methods. Results: No differences were found in models scanned at different angulations. Better superimposition correlation was observed between methods 3 and 4 those used techniques based on palatal surface associated to anatomical landmarks to superimpose. Conclusions: Linear measurements and superimpositions were not affected by the different angulations of the models during the scanning process. The most reliable superimposition methods were the ones using surface and landmarks.
\end{abstract}

\section{Keywords}

Dental Models, Dental Scanner, Three-Dimensional, Orthodontics, Palate 


\section{Introduction}

Successful orthodontic treatment is the one in which the objectives of treatment are attained and maintained over the years. In order to achieve this, an individual and extensive diagnosis and treatment plan should be done. This is carried out based on appropriate records, consisting of radiographs, photographs and study models. Dental models play an important role in this, being crucial to assess intercanine and intermolar widths, space availability, irregularity index, Bolton analysis, overbite, overjet, tooth size and arch length [1] [2]. However, plaster models are subject to loss, fracture, and degradation; besides being difficult to share. Advancements in virtual technologies have increased the evaluation possibilities using digital models. They can be obtained from dental casts or impressions with a desktop scanner, or digitalized directly from the patients' teeth using an intraoral scanner, eliminating the conventional impression step. These contemporary acquisition methods are adequate for orthodontic diagnosis and treatment planning.

Careful evaluation of the orthodontic tooth movement is crucial for understanding the dental biomechanics. The superimposition of serial cephalograms has been widely used for dentoskeletal evaluation in orthodontics. However, cephalometric superimpositions have several limitations, including tracing errors, radiation exposure, and difficulties in evaluating two-dimensional (2D) projection of three-dimensional (3D) structures. Researchers are investigating the accuracy of measurements using digital models through different softwares comparing the results to plaster models, without significant differences among them [1]-[9]. One advantage of the digital models is the possibility of superimposing them. Using specific software programs available for image processing of stereolithography (.stl) files, dental casts can also be superimposed at different treatment stages. Thus, the Dentist can evaluate dental movement and skeletal changes in the 3 dimensions $(x, y, z)$, so that the professional may see if the results are compatible with the original planning, any side effects and compare different orthodontic mechanics, among other reasons [10] [11] [12] [13] [14]. This kind of evaluation requires the use of stable structures as references for superimposition, which according to the literature should be the first three palatal rugae and the area of the palatal vault [11] [12] [13] [14] [15]. However, a collection of thousands of points coordinated in a 3D space and oriented as polygon meshes are obtained after scanning when questions can be raised, such as: can the dimensional surfaces of the digital models be influenced by a different positioning of the dental casts during the scanning process? Can these .stl files be used with accuracy for registration and superimposition of digital models?

The aims of this study were to assess and compare the linear measurements in digital models, scanned at four different angulations, to compare four methods of superimposing digital models.

\section{Material and Methods}

\section{Sample Selection}


This study was approved by the Ethical Committee of Pontifical Catholic University of Rio Grande do Sul (PUCRS) (CAAE: 42856915.1.0000.5336). The sample of this study comprised of 43 upper dental casts of young patients (27 female, 16 male) obtained at the start of orthodontic treatment, as described in Table 1. The sample size was calculated before the study. It was estimated that a sample size of 43 dental models would be necessary to obtain a statistical power of $95 \%$.

\section{Procedures Description}

The dental casts were scanned using a 3D desktop scanner R700 (3Shape, Copenhagen, Denmark), creating virtual dental models. The images were processed and evaluated using OrthoAnalyzer Software (3Shape, Copenhagen, Denmark). Each dental model was acquired at a different angulation (0, 5, 10 and 15 degrees in relation to the occlusal plane) divided in 4 groups (named as $0,5,10$ and 15). This model angulation was standardized with acrylic platforms produced with the mentioned angulations, in which the models were positioned during the scanning process (Figure 1). The angulations of 0 to 15 degrees were selected for covering most malocclusions, involving an asymmetric or canted occlusal plane [16].

\section{Linear Measurements Definition}

A total of 18 linear measurements were made to evaluate the central and the lateral parts of the models (Table 2, Figure 2). Besides that, the four groups were compared to detect possible alterations or distortions due to models different angulations during the scanning process.

\section{Evaluation of the Superimposition Methods}

The scanned 0 and 15 degrees stl files, representing the original and the most reoriented model, were superimposed using four overlapping methods (Figure 3). The digital model superimposition was performed using three landmarks (Methods 1 and 2); one landmark + surface (Method 3); and three landmarks + surface (Method 4) (Table 3). In the present study, the palatal rugae were chosen as the central points, once they are considered stable references in the literature [1] [11] [15]. Other selected points were located in the upper models lateral

Table 1. Sample selection criteria.

\begin{tabular}{c} 
Inclusion criteria \\
\hline Permanent dentition (presence of all teeth in the arch, until the second molars) \\
High quality dental casts \\
Good visualization of the palatal rugae area \\
Exclusion criteria \\
Fractures or bubbles \\
Absence of one or more teeth (excluding the third molars) \\
Ectopic teeth or anomalies in tooth shape \\
Cleft lip and palate or syndromic patients
\end{tabular}


Table 2. Description of the variables measured in digital models.

\begin{tabular}{|c|c|c|}
\hline Landmarks & Variables & Description \\
\hline 1 & \multirow{2}{*}{ LR1R LR1L } & Length of the right first ruga \\
\hline 2 & & Length of the left first ruga \\
\hline 3 & LR3R & Length of the right third ruga \\
\hline 4 & LR3L & Length of the left third ruga \\
\hline 5 & DM1 & $\begin{array}{l}\text { Transverse linear distance between the medial points of the right and } \\
\text { left first rugae }\end{array}$ \\
\hline 6 & DM3 & $\begin{array}{l}\text { Transverse linear distance between the medial points of the right and } \\
\text { left third rugae }\end{array}$ \\
\hline 7 & DL1 & $\begin{array}{l}\text { Transverse linear distance between the lateral points of the right and } \\
\text { left first rugae }\end{array}$ \\
\hline 8 & DL3 & $\begin{array}{l}\text { Transverse linear distance between the lateral points of the right and } \\
\text { left third rugae }\end{array}$ \\
\hline 9 & APMR13 & $\begin{array}{l}\text { Anteroposterior distance between the medial points of the right first } \\
\text { and third rugae }\end{array}$ \\
\hline 10 & APML13 & $\begin{array}{l}\text { Anteroposterior distance between the medial points of the left first and } \\
\text { third rugae }\end{array}$ \\
\hline 11 & APLR13 & $\begin{array}{l}\text { Anteroposterior distance between the lateral points of the right first and } \\
\text { third rugae }\end{array}$ \\
\hline 12 & APLL13 & $\begin{array}{l}\text { Anteroposterior distance between the lateral points of the left first and } \\
\text { third rugae }\end{array}$ \\
\hline 13 & $\mathrm{C}-\mathrm{C}$ & Distance between the occlusal tips of the canines \\
\hline 14 & M-M & Distance between the mesio-buccal cusps of the first permanent molar \\
\hline 15 & CR-MR & $\begin{array}{l}\text { Distance of tips of the right canine and mesio-buccal cusp of first } \\
\text { permanent molar }\end{array}$ \\
\hline 16 & CL-ML & $\begin{array}{l}\text { Distance of tips of the left canine and mesio-buccal cusp of first } \\
\text { permanent molar }\end{array}$ \\
\hline 17 & CR-ML & $\begin{array}{l}\text { Distance of tips of the right canine and mesio-buccal cusp of left first } \\
\text { permanent molar }\end{array}$ \\
\hline 18 & CL-MR & $\begin{array}{l}\text { Distance of tips of the left canine and mesio-buccal cusp of right first } \\
\text { permanent molar }\end{array}$ \\
\hline
\end{tabular}

Table 3. Description of the four superimposition methods evaluated and its anatomical references.

\begin{tabular}{|c|c|c|c|}
\hline Method & $\begin{array}{l}\text { Superimposition } \\
\text { landmarks }\end{array}$ & Description & Example \\
\hline 1 & $\begin{array}{l}\text { Three central } \\
\text { landmarks }\end{array}$ & $\begin{array}{l}\text { The medial point of the right } \\
\text { second palatal ruga, and the } \\
\text { medial points of the right and left } \\
\text { third rugae }\end{array}$ & \\
\hline
\end{tabular}




\section{Continued}

Three lateral landmarks Surface + one landmark

4 Surface + three landmarks
Occlusal tip of the right canine, buccal cusp of the left first premolar and mesio-buccal cusp of the right first permanent molar

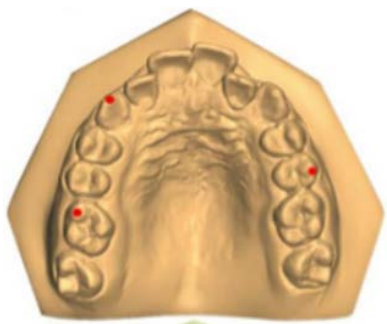

The medial point of the right third ruga and the surface selected on the palate

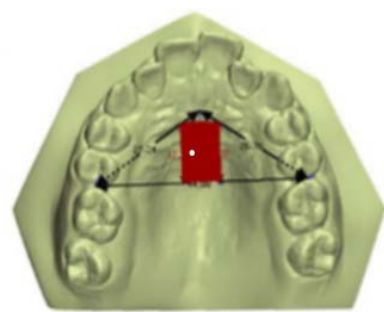

The medial point of the right second palatal ruga, the medial points of the right and left third rugae, and the surface selected on the palate

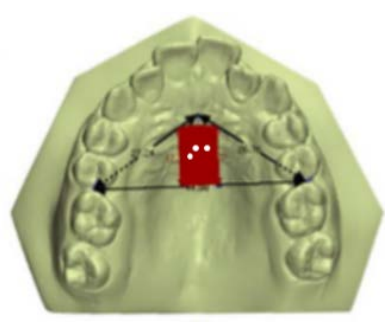

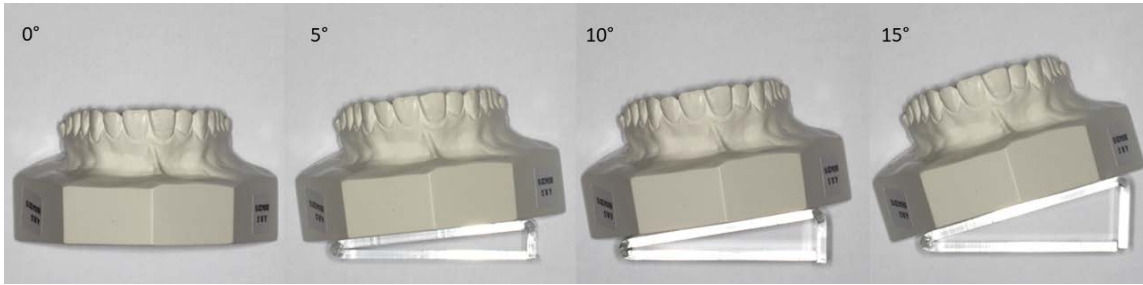

Figure 1. Study models positioned on acrylic platforms for scanning at 0, 5, 10 and 15 degrees in relation to the occlusal plane.

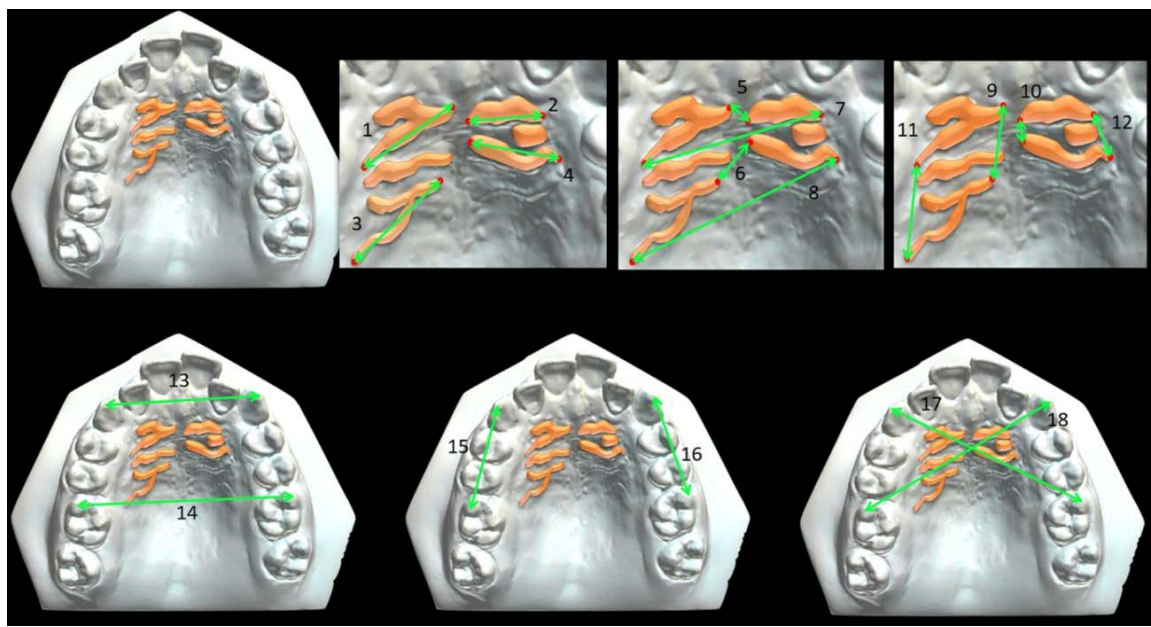

Figure 2. Digital models showing the landmarks (1 to 18 ) located at the palatine rugae region and teeth used as references for the dimensional evaluation. 
regions, as the right canine (cusp tip), the left second premolar (buccal cusp tip) and the right first molar (mesial buccal cusp tip) (Figure 3(C) and Figure 3(D)). These points were used to superimpose the digital models in Methods 1 and 2. In Methods 3 and 4, apart from the central points, a surface was selected to superimpose the digital models. This surface was determined by a triangle formed by a straight line connecting the mesial surface of the right and left first molars,
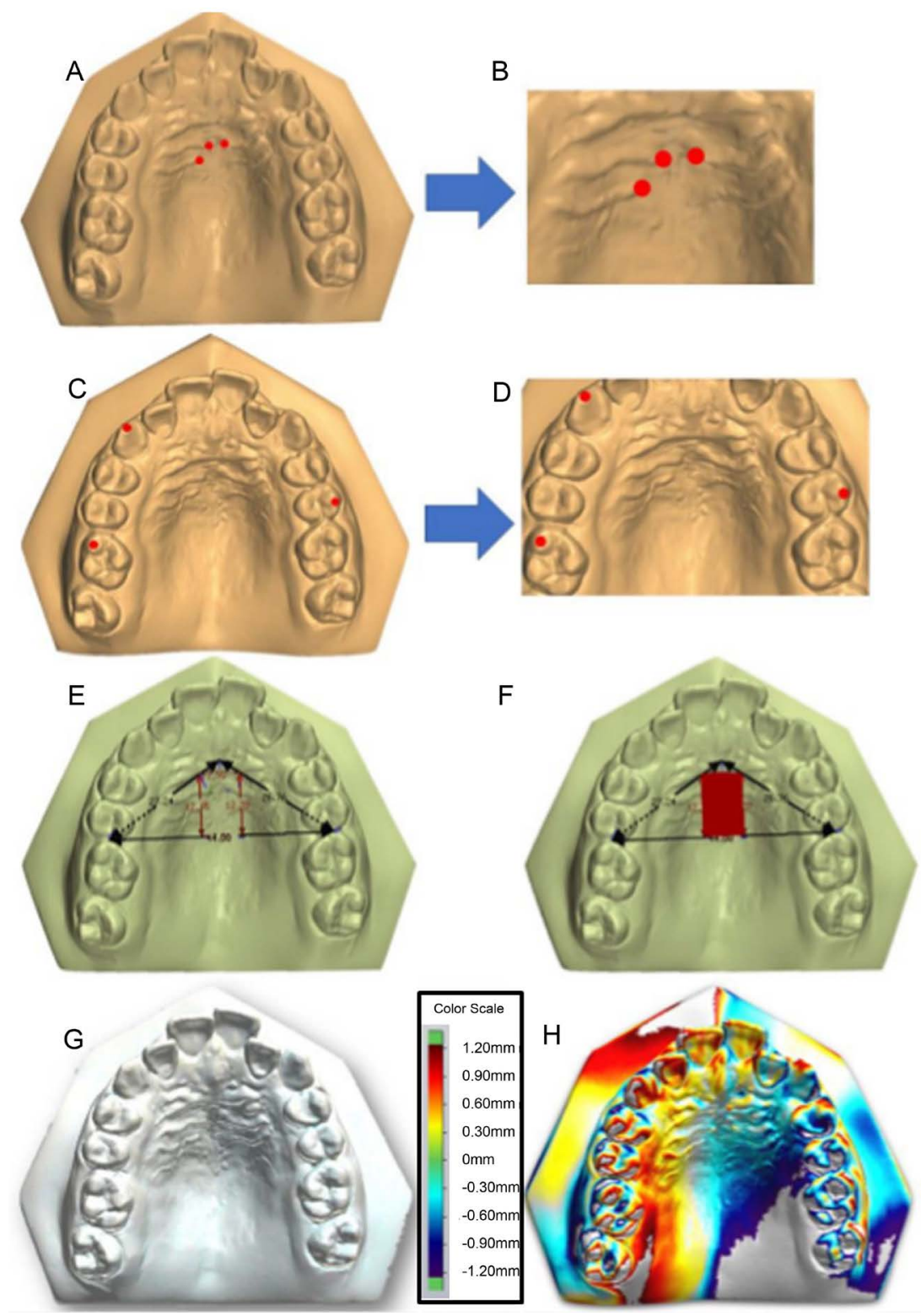

Figure 3. Four superimposition methods evaluated. Method 1) Central landmarks at the palatal rugae region [A]; and magnified view [B]. Method 2) Three lateral landmarks positioned at the occlusal tip of the right canine, buccal cusp of the left first premolar and mesio-bucal cusp of the right first permanent molar [C]; and magnified view [D]. Method 3) Surface + one landmark using as anatomical references the medial point of the right third ruga and a rectangular area of palatal surface [E]; Method 4) The same palatal surface than in method 3, but three palatal landmarks are associated than in the method 1 [F]. Color map and superimposed models showing high [G] and slight reproducibility $[\mathrm{H}]$. 
plus, a line connecting the most posterior point of the incisive papilla to the limit of the line between the right and left first molars (Figure 3(E) and Figure 3(F)). Then, a rectangular area was delineated inside this triangle whose lateral limits were $4 \mathrm{~mm}$ to each side of the palatine raphe, using the posterior limit of the triangle. From this straight line, a 90 degrees angle was formed at both lateral limits. The anterior limit of the rectangle was where the right and left lateral limits of the triangle and the rectangle met. The most medial point of the first three right and left palatal rugae and a part of the palatal vault were located in this area. The selected surface used for superimposing the models is shown in red, in Figure 3(F).

Color maps were used to evaluate the reproducibility of the superimpositions, performed by overlapping each of the 43 models scanned at 0 and 15 degrees. The best fit of the models was evaluated at $0.3 \mathrm{~mm}$. High reproducibility (A) was considered when the difference ranged from $-0.3 \mathrm{~mm}$ to $0.3 \mathrm{~mm}$, and slight reproducibility (B) when the difference was above or below this range (Figure $3(\mathrm{G})$ and Figure $3(\mathrm{H})$, respectively). Hence, the color maps were calibrated at $0.3 \mathrm{~mm}$, positive and negative. So, dental and palatal surface variance was represented by color progression from blue to purple when the difference was negative, and from yellow to dark red when the difference was positive.

\section{Statistical Analysis}

Double assessments of all measures, in a two-week interval, were done by the same examiner (B.B.), previously trained. Intra-observer reliability and reproducibility were evaluated using the Intraclass Correlation Coefficient (ICC). The Shapiro-Wilk test was used to check the normality of the data, which showed a non-normal distribution. Hence, nonparametric tests were applied. Kruskal-Wallis test was used to compare the median values of the measurements of groups. Kappa coefficient was used to evaluate the agreement on the superimposition of the digital models at 0 and 15 degrees. All statistical analyses were carried out with R version 3.4.1 (R Core Team 2017, Vienna, Austria).

\section{Results}

Reproducibility as well as reliability of the observer showed excellent agreement (ICC > 0.921). ICC to compare the groups showed near perfect correlation (ICC > $0.962)$.

All linear measurements are presented in Table 4. By Kruskal-Wallis test none of the linear measurements presented statistical difference. The median differences between the groups $(0,5,10$ and 15) ranged from $0.03 \mathrm{~mm}$ to 0.39 $\mathrm{mm}$.

Color maps calibrated to detect differences up to $0.3 \mathrm{~mm}$ were used to compare the model superimposition methods. Method 1 (central landmarks) showed 3 superimpositions with a high correlation and 40 with a slight correlation. Method 2 (lateral landmarks) showed 36 superimpositions with a high correlation and 7 with a slight correlation. Method 3 (surface + one landmark) and 4 (surface + three landmarks) showed 39 superimpositions with a high correlation and 
Table 4. Comparison of linear measurements of the four groups (Kruskal-Wallis).

\begin{tabular}{|c|c|c|c|c|c|c|c|c|c|c|c|c|c|c|}
\hline \multirow{2}{*}{$\begin{array}{l}\text { Variables } \\
(\mathrm{mm})\end{array}$} & \multicolumn{3}{|c|}{$0^{\circ}$} & \multicolumn{3}{|c|}{$5^{\circ}$} & \multicolumn{3}{|c|}{$10^{\circ}$} & \multicolumn{3}{|c|}{$15^{\circ}$} & \multicolumn{2}{|c|}{ Group Difference } \\
\hline & Mean & Med & sd & Mean & Med & sd & Mean & Med & sd & Mean & Med & sd & $\mathrm{K}$ & $\rho^{*}$ \\
\hline LR1R & 9.05 & 9.85 & 1.98 & 9.07 & 9.88 & 1.98 & 9.07 & 10.05 & 1.99 & 9.09 & 10.08 & 1.98 & 0.029 & 0.999 \\
\hline LR1L & 9.30 & 9.92 & 1.50 & 9.30 & 10.04 & 1.52 & 9.35 & 9.86 & 1.52 & 9.30 & 9.94 & 1.52 & 0.010 & 0.999 \\
\hline LR3R & 8.81 & 9.15 & 3.69 & 8.86 & 9.26 & 3.71 & 8.84 & 9.17 & 3.74 & 8.84 & 9.32 & 3.69 & 0.022 & 0.999 \\
\hline LR3L & 8.09 & 7.69 & 3.64 & 8.05 & 7.72 & 3.59 & 8.05 & 7.71 & 3.59 & 8.05 & 7.65 & 3.52 & 0.008 & 0.999 \\
\hline DM1 & 2.95 & 3.02 & 1.69 & 3.00 & 3.16 & 1.63 & 2.93 & 2.94 & 1.73 & 2.88 & 2.90 & 1.69 & 0.101 & 0.991 \\
\hline DM3 & 9.02 & 9.39 & 3.71 & 8.91 & 9.40 & 3.78 & 8.93 & 9.18 & 3.76 & 8.91 & 9.04 & 3.75 & 0.015 & 0.999 \\
\hline DL1 & 18.30 & 18.71 & 2.59 & 18.28 & 18.98 & 2.57 & 18.23 & 18.86 & 2.50 & 18.35 & 19.10 & 2.54 & 0.065 & 0.996 \\
\hline DL3 & 20.86 & 20.93 & 2.71 & 20.98 & 20.92 & 2.76 & 20.86 & 20.86 & 2.73 & 20.91 & 20.94 & 2.69 & 0.023 & 0.999 \\
\hline APMR13 & 8.65 & 8.95 & 3.34 & 8.72 & 9.11 & 3.34 & 8.67 & 8.90 & 3.32 & 8.67 & 8.95 & 3.24 & 0.055 & 0.997 \\
\hline APML13 & 7.12 & 7.58 & 2.54 & 7.07 & 7.47 & 2.49 & 7.12 & 7.43 & 2.48 & 7.12 & 7.40 & 2.48 & 0.058 & 0.996 \\
\hline APLR13 & 9.07 & 9.52 & 3.20 & 9.12 & 9.50 & 3.15 & 9.09 & 9.63 & 3.15 & 9.07 & 9.71 & 3.21 & 0.017 & 0.999 \\
\hline APLL13 & 6.24 & 6.08 & 2.35 & 6.24 & 6.10 & 2.35 & 6.24 & 6.08 & 2.34 & 6.23 & 6.11 & 2.36 & 0.006 & 0.999 \\
\hline C-C & 32.65 & 33.35 & 3.05 & 32.67 & 33.18 & 2.30 & 32.65 & 33.36 & 2.98 & 32.58 & 33.32 & 2.95 & 0.01 & 0.999 \\
\hline M-M & 48.88 & 49.45 & 2.94 & 48.93 & 49.53 & 3.00 & 48.95 & 49.60 & 3.00 & 48.95 & 49.57 & 3.06 & 0.002 & 0.999 \\
\hline CR-MR & 20.91 & 22.59 & 3.63 & 20.86 & 22.64 & 3.66 & 20.91 & 22.55 & 3.70 & 20.93 & 22.59 & 3.75 & 0.037 & 0.998 \\
\hline CL-ML & 21.07 & 22.28 & 3.66 & 20.91 & 22.30 & 3.64 & 21.00 & 22.45 & 3.65 & 20.98 & 22.13 & 3.65 & 0.073 & 0.994 \\
\hline CR-ML & 45.63 & 46.16 & 2.71 & 45.63 & 46.04 & 2.77 & 45.65 & 46.17 & 2.66 & 45.58 & 46.10 & 2.68 & 0.022 & 0.999 \\
\hline CL-MR & 45.12 & 45.64 & 2.75 & 45.14 & 45.57 & 2.84 & 45.09 & 45.42 & 2.80 & 45.09 & 45.52 & 2.80 & 0.018 & 0.999 \\
\hline
\end{tabular}

only 4 with a slight correlation, demonstrating excellent accuracy for model superimposition (Table 5).

The agreement evaluation using Kappa coefficient among the superimposition methods showed high reproducibility between Methods 3 - 4 and a substantial agreement between Methods $2-3$ and 2 - 4. Method 1 showed a fair agreement with Method 2, and a slight agreement with Methods 3 and 4, as observed in Table 6.

\section{Discussion}

Different software programs are able to evaluate digital models during orthodontic diagnosis and treatment planning, with recent technological advances in dentistry [1]-[8]. Variations lower than $0.5 \mathrm{~mm}$ between plaster and digital models are considered clinically acceptable [2] [3] [4] [5] [16] [17] [18]. However, Noh et al. [19] showed that discrepancies from 0.27 to $0.33 \mathrm{~mm}$ could lead to errors in the manufacturing of orthodontic appliances and surgical guides. The present study evaluated the hypothesis that the acquisition of the same models scanned at different angulations could show alterations, with a variation of the linear measurements. In addition, the 15 degrees was chosen because, according to Birgfeld et al. [16] this level of angulation comprises most of the malocclusion 
Table 5. Reproducibility of the different methods of models superimposition.

\begin{tabular}{ccccc}
\hline & Method 1 & Method 2 & Method 3 & Method 4 \\
\hline A & 3 & 36 & 39 & 39 \\
B & 40 & 7 & 4 & 4 \\
\hline
\end{tabular}

(A) High reproducibility; (B) Slight reproducibility.

Table 6. Evaluation of agreement between superimposition methods.

\begin{tabular}{cccc}
\hline $\begin{array}{c}\text { Agreement between superimposi- } \\
\text { tion methods }\end{array}$ & $(\mathrm{mm})$ & $\mathrm{K} *$ & $\mathrm{p}$ \\
\hline 1 and 2 & 0.3 & 0.029 & 0.428 \\
1 and 3 & 0.3 & 0.015 & 0.565 \\
1 and 4 & 0.3 & 0.015 & 0.565 \\
2 and 3 & 0.3 & $\mathbf{0 . 6 9 1}$ & $<0.001^{\star}$ \\
2 and 4 & 0.3 & $\mathbf{0 . 6 9 1}$ & $<0.001^{\star}$ \\
3 and 4 & 0.3 & 1.000 & $<0.001^{\star}$ \\
\hline
\end{tabular}

Significant $(\mathrm{p}<0.05)^{*}$; Kappa coefficient ${ }^{* *}$.

cases with the cant of the occlusal plane, as well as physical problems during the plaster models trimming or scanning process. Eighteen landmarks in both central and lateral parts of the model were selected for measurements so that any possible distortion would be detected. The results of the linear measurements showed excellent agreement (Table 6), with an average difference among the groups ranging from $0.03 \mathrm{~mm}$ to $0.39 \mathrm{~mm}$. A difference higher than 0.33 at the rugae measures was observed (DM3 and DL1) which could cause a poor adaptation of the orthodontic aligners and the surgical guides. Although when comparing pre and post treatment results (follow-up), these differences might not be considered significant. This means that the digital models scanned at angulations between 0 and 15 degrees did not show significant alterations to the point of causing changes in evaluations such as diagnosis and treatment planning, or comparisons between different phases of the orthodontic treatment.

Four methods of superimposition were used to evaluate the correlation between the digital models scanned at 0 and 15 degrees (original and reoriented models) (Figure 3). Methods 3 (surface + one landmark) and 4 (surface + three landmarks) resulted in more than $90 \%$ of correlation between the surfaces with the color maps calibrated at $0.3 \mathrm{~mm}$. This high correlation is probably because they presented a selected surface area (surface-to-surface matching), besides the points, to assist in the superimposition. Method 2 (three lateral landmarks) was selected to test the overlapping with three distant points. This method presented more than $83 \%$ of correlation, most likely due to the greater distance among the points, decreasing the rotational error at the moment of the superimposition, compared to Method 1. There was a slight correlation in all superimpositions in Method 1 (three central landmarks), where the three points were selected near 
each other. This could have happened because the points were too close and did not have a surface to assist with the superimposition. So, there was a rotational error when the superimposition was performed, which is in agreement with Chen et al. [11] and Jang et al. [15], proving this method to be unreliable for superimposition purposes (Table 5). The agreement evaluation by Kappa coefficient among the superimposition methods showed that the two most accurate methods were 3 and 4 . They also presented the highest correlation among them, indicating the need of a surface-to-surface matching to achieve an almost perfect superimposition (Table 6).

Areas that suffer the least modification during orthodontic treatment should be used to perform accurate model superimposition. Until the present moment, the most stable anatomic structures in the maxilla are considered to be the first three palatal rugae for a short period of time difference [11] [15] [20] [21]. After 40 years, these structures are lower than acceptable for overlapping [21].

Changes in palatal shape may be a source of error in the 3D superimposition method [12] depending on the regions selected for overlapping. What should determine the landmarks choice to superimpose are the regions of the palate that suffer the least modification with dental movement during treatment. Standardizing the superimposition in the present study, apart from using the same model, with no dental modification, an area in the center of the palate was selected, including the most medial points of the first three palatal rugae on both sides and a part of the palatal vault. All these reference landmarks have been considered stable until now; although they should be used carefully since new studies are being conducted, with new technologies that can change some existing concepts.

Digital model studies are interesting and becoming the gold standard as technology is becoming accessible to all. Diagnoses, treatment planning and orthodontic result evaluation processes are nowadays mostly virtual. One of the methods to access the orthodontic results is the superimposition. In the orthodontic literature there are different kinds of superimposition, however, a question that has come up lately is: is the appropriate method being used? According to this study, the ideal superimposition would be using a surface and a point simultaneously. Another important question is the model angulation during 3D scanning - can the dimensional surfaces of the digital models be influenced by a different positioning of the dental casts during the scanning process? This research showed that scanned models with angulations between $0^{\circ}$ and $15^{\circ}$ did not present a significant difference in the obtained measurements. However, further researches are needed to evaluate stable regions to perform superimposition of digital models with accuracy, on specific situations and procedures.

\section{Conclusions}

The scanning angulations of digital models did not affect the linear measurements and superimpositions. 
The most reliable digital model superimposition methods were the ones using surface and landmarks simultaneously.

\section{Acknowledgements}

This study was financed in part by the Coordenaçãode Aperfeiçoamento de Pessoal de Nível Superior-Brasil (CAPES)—FinanceCode 001.

\section{Conflicts of Interest}

The authors declare no conflicts of interest regarding the publication of this paper.

\section{References}

[1] Horton, H.M.I., Miller, J.R., Gaillard, P.R. and Larson, B.E. (2010) Technique Comparison for Efficient Orthodontic Tooth Measurements Using Digital Models. The Angle Orthodontist, 80, 254-261. https://doi.org/10.2319/041709-219.1

[2] Lippold, C., Kirschneck, C., Schreiber, K., et al. (2015) Methodological Accuracy of Digital and Manual Model Analysis in Orthodontics-A Retrospective Clinical Study. Computers in Biology and Medicine, 62, 103-109. https://doi.org/10.1016/j.compbiomed.2015.04.012

[3] Abizadeh, N., Moles, D.R., O’Neill, J. and Noar, J.H. (2012) Digital versus Plaster Study Models: How Accurate and Reproducible Are They? Journal of Orthodontics, 39, 151-159. https://doi.org/10.1179/1465312512Z.00000000023

[4] Leifert, M.F., Leifert, M.M., Efstratiadis, S.S. and Cangialosi, T.J. (2009) Comparison of Space Analysis Evaluations with Digital Models and Plaster Dental Casts. American Journal of Orthodontics and Dentofacial Orthopedics, 136, 16.e1-16.e4. https://doi.org/10.1016/j.ajodo.2008.11.019

[5] Sousa, M.V.S., Vasconcelos, E.C., Janson, G., Garib, D. and Pinzan, A. (2012) Accuracy and Reproducibility of 3-Dimensional Digital Model Measurements. American Journal of Orthodontics and Dentofacial Orthopedics, 142, 269-273. https://doi.org/10.1016/j.ajodo.2011.12.028

[6] Rheude, B., Sadowsky, P.L., Ferriera, A. and Jacobson, A. (2005) An Evaluation of the Use of Digital Study Models in Orthodontic Diagnosis and Treatment Planning. The Angle Orthodontist, 75, 300-304.

[7] Whetten, J.L., Williamson, P.C., Heo, G., Varnhagen, C. and Major, P.W. (2006) Variations in Orthodontic Treatment Planning Decisions of Class II Patients between Virtual 3-Dimensional Models and Traditional Plaster Study Models. American Journal of Orthodontics and Dentofacial Orthopedics, 130, 485-491. https://doi.org/10.1016/j.ajodo.2005.02.022

[8] Pachêco-Pereira, C., de Luca Canto, G., Major, P.W. and Flores-Mir, C. (2015) Variation of Orthodontic Treatment Decision-Making Based on Dental Model Type: A Systematic Review. The Angle Orthodontist, 85, 501-509.

https://doi.org/10.2319/051214-343.1

[9] Kim, J. and Lagravére, M.O. (2016) Accuracy of Bolton Analysis Measured in Laser Scanned Digital Models Compared with Plaster Models (Gold Standard) and Cone-Beam Computer Tomography Images. Korean Journal of Orthodontics, 46, 13-19. https://doi.org/10.4041/kjod.2016.46.1.13

[10] Akyalcin, S., Dyer, D.J., English, J.D. and Sar, C. (2013) Comparison of 3-Dimensional 
Dental Models from Different Sources: Diagnostic Accuracy and Surface Registration Analysis. American Journal of Orthodontics and Dentofacial Orthopedics, 144, 831-837. https://doi.org/10.1016/j.ajodo.2013.08.014

[11] Chen, G., Chen, S., Zhang, X., et al. (2011) Stable Region for Maxillary Dental Cast Superimposition in Adults, Studied with the Aid of Stable Miniscrews. Orthodontics and Craniofacial Research, 14, 70-79. https://doi.org/10.1111/j.1601-6343.2011.01510.x

[12] Choi, J.-I., Cha, B.-K., Jost-Brinkmannm, P.-G., Choim, D.-S. and Jangm, I.-S. (2012) Validity of Palatal Superimposition of 3-Dimensional Digital Models in Cases Treated with Rapid Maxillary Expansion and Maxillary Protraction Headgear. Korean Journal of Orthodontics, 42, 235-241. https://doi.org/10.4041/kjod.2012.42.5.235

[13] Nalcaci, R., Kocoglu-Altan, A.B., Bicakci, A.A., Ozturk, F. and Babacan, H. (2015) A Reliable Method for Evaluating Upper Molar Distalization: Superimposition of Three-Dimensional Digital Models. Korean Journal of Orthodontics, 45, 82-88. https://doi.org/10.4041/kjod.2015.45.2.82

[14] Elnagar, M.H., Elshourbagy, E., Ghobashy, S., Khedr, M. and Evans, C.A. (2017) Dentoalveolar and Arch Dimension Changes in Patients Treated with Miniplate-Anchored Maxillary Protraction. American Journal of Orthodontics and Dentofacial Orthopedics, 151, 1092-1106. https://doi.org/10.1016/j.ajodo.2016.10.038

[15] Jang, I., Tanaka, M., Koga, Y., et al. (2009) A Novel Method for the Assessment of Three-Dimensional Tooth Movement during Orthodontic Treatment. The Angle Orthodontics, 79, 447-453. https://doi.org/10.2319/042308-225.1

[16] Birgfeld, C.B., Saltzman, B.S., Luquetti, D.V., Latham, K., Starr, J.R. and Heike, C.L. (2013) Comparison of Two-Dimensional and Three-Dimensional Images for Phenotypic Assessment of Craniofacial Microsomia. The Cleft Palate and Craniofacial Journal, 50, 305-314. https://doi.org/10.1597/11-173

[17] Fleming, P., Marinho, V. and Johal, A. (2011) Orthodontic Measurements on Digital Study Models Compared with Plaster Models: A Systematic Review. Orthodontics and Craniofacial Research, 14, 1-16. https://doi.org/10.1111/j.1601-6343.2010.01503.x

[18] de Luca Canto, G., Pachêco-Pereira, C., Lagravere, M.O., Flores-Mir, C. and Major, P.W. (2015) Intra-Arch Dimensional Measurement Validity of Laser-Scanned Digital Dental Models Compared with the Original Plaster Models: A Systematic Review. Orthodontics and Craniofacial Research, 18, 65-76.

https://doi.org/10.1111/ocr.12068

[19] Noh, H., Nabha, W., Cho, J.-H. and Hwang, H.-S. (2011) Registration Accuracy in the Integration of Laser-Scanned Dental Images into Maxillofacial Cone-Beam Computed Tomography Images. American Journal of Orthodontics and Dentofacial Orthopedics, 140, 585-591. https://doi.org/10.1016/j.ajodo.2011.04.018

[20] Cha, B.K., Lee, J.Y., Jost-Brinkmann, P.-G. and Yoshida, N. (2007) Analysis of Tooth Movement in Extraction Cases Using Three-Dimensional Reverse Engineering Technology. European Journal of Orthodontics, 29, 325-331. https://doi.org/10.1093/ejo/cjm019

[21] Garib, D., Miranda, F., Yatabe, M.S., et al. (2019) Superimposition of Maxillary Digital Models Using the Palatal Rugae: Does Ageing Affect the Reliability? Orthodontics and Craniofacial Research, 22, 183-193. https://doi.org/10.1111/ocr.12309 